\title{
Investigating cyclist interaction behavior through a controlled laboratory experiment
}

\author{
Yufei Yuan (Corresponding author) \\ Delft University of Technology \\ y.yuan@tudelft.nl \\ Bernat Goñi-Ros \\ Delft University of Technology \\ b.goniros@tudelft.nl
}

\author{
Winnie Daamen \\ Delft University of Technology \\ w.daamen@tudelft.nl \\ Serge P. Hoogendoorn \\ Delft University of Technology \\ s.p.hoogendoorn@tudelft.nl
}

\begin{abstract}
Nowadays, there is a need for tools to support city planners in assessing the performance of cycling infrastructure and managing bicycles and mixed flows. Microscopic and macroscopic bicycle traffic models can be used to fulfill this need. However, fundamental knowledge on individual cyclist interaction behavior (which should underpin these models) is hardly available in literature. Detailed bicycle traffic data are necessary if we want to gain insight into cyclist interaction behavior and develop sound behavioral theories and models. Laboratory experiments have been proven to be one of the most effective ways to collect detailed traffic data. For this reason, a controlled experiment aimed to investigate cyclist interaction behavior has been carried out at Delft University of Technology. This paper describes the experimental design, the resulting microscopic bicycle trajectories, and some preliminary results regarding one of the most common interaction situations: the bidirectional interaction. The preliminary results reveal how and to what extent cyclists interact in bidirectional cycling. It is found that cyclists perform a clearly-visible evading (collision avoidance) maneuver when they have face-to-face encounters. During these maneuvers, changes in speed and displacements in the lateral direction are observed. Cyclists start to deviate from their original path when they are around $30 \mathrm{~m}$ from each other, and they strongly prefer passing on the right-hand side. Moreover, the expectation of gender differences in cycling behavior reported in the literature is confirmed: our results show that women generally cycle more slowly than men and deviate more from their intended paths in face-to-face encounters. More observations will be available in the next stage of data analysis. These findings can be used to formulate improved microscopic bicycle traffic models for infrastructure design and policy development.
\end{abstract}

\section{Article history:}

Received: February 15, 2017

Received in revised form:

November 1, 2017

Accepted: April 13, 2018

Available online: October 22, 2018

Keywords: controlled laboratory experiment, cyclist interaction behavior, bicycle trajectory data, bidirectional flows

Copyright 2018 Yufei Yuan, Winnie Daamen, Bernat Gońi-Ros \& Serge Hoogendoorn http://dx.doi.org/10.5198/jtlu.2018.1155

ISSN: 1938-7849 | Licensed under the Creative Commons Attribution - Noncommercial License 4.0

The Journal of Transport and Land Use is the official journal of the World Society for Transport and Land Use (WSTLUR) and is published and sponsored by the University of Minnesota Center for Transportation Studies. 


\section{Introduction}

In big cities, the proportion of "active mode" traffic (such as pedestrians and bicyclists) in the total transport trip demand is steadily growing every year (Amsterdam-Municipality, 2011). Although there are many reasons to welcome this trend, various concerns arise with respect to crowdedness and safety in big events, shopping areas, and shared space areas where mixed flows interact. To assess the performance of cycling infrastructure and to support the planning and management of bicycle and mixed flows in an urban context, tools to aid city planners and policy makers are required. In addition to design guidelines, microscopic and macroscopic bicycle traffic models can be used to fulfill this need. In literature, fundamental knowledge on individual cyclist interaction behavior (the managing of conflicts with other cyclists) underpinning these models is hardly available. Neither the heterogeneity in cycling behavior, nor the difference in behavior in different interaction situations is covered in the existing (bicycle) traffic flow theories. Most existing research on cycling behavior focuses on quantifying macroscopic characteristics of bicycle traffic flow, such as free speed, critical density, capacity and jam density (Botma \& Papendrecht, 1991; Gould \& Karner, 2009; Homburger, 1976; Hoogendoorn \& Daamen, 2016; Navin, 1994; Raksuntorn \& Khan, 2003; Seriani, Fernandez, \& Hermosilla, 2015; Yang, 1985; Zamanov, 2012). The main goal of this research is to identify the effect of interaction between cyclists on individual cycling behavior. To this end, detailed bicycle flow data (e.g., microscopic trajectory data) are required to get insight into cyclist behavior, and further to test and develop behavioral theories, as well as to verify, calibrate and validate simulation models.

These data can be obtained either from real-world observations or from laboratory experiments. Although participants behave in their natural circumstances during real-world observations, many unexpected (external) conditions may occur. And those conditions are difficult to be controlled, so the circumstances of the measurements are not always under the same conditions. More importantly, the effect of these external conditions, the heterogeneity of the population and the intra-variability of individual behavior, cannot be identified accurately. Even if the latter is not the case, this may lead to a long observation period when observation takes place in practice under certain conditions. Therefore, laboratory experiments in controlled conditions can be considered to collect cyclist flow data. This type of experiments does not only leave out external conditions, but it also keeps the conditions constant for all observations. The experiments then can easily be repeated in the same circumstances and various specific situations can be produced and tested out. Furthermore, the monitoring will be easier, as the site can be chosen such that monitoring requirements are met.

There are many existing laboratory experiments to study the behavior of other modes, such as pedestrians and car traffic flows. For instance, Daamen and Hoogendoorn (2003) performed an experimental study on pedestrian walking behavior where different types of walking and interactions were investigated. Huber, et al. (2014) designed an experiment to study how a pedestrian adjusts its speed and path when avoiding collisions with another pedestrian. Seyfried, Steffen, Passon, and Klingsch (2009) studied the pedestrian flow capacity through bottlenecks under laboratory conditions. Tian, Ma, Song, and Liddle (2012) designed an experiment to see how pedestrians evacuated from corridors of different widths. Moussaïd et al. (2009) performed an experiment to investigate pedestrian interactions in a corridor. Daamen, Hoogendoorn, Campanella, and Versluis (2012) further extended the study on pedestrian interactions in a more generic context. Also, a circuit experiment was performed to investigate shock-wave phenomena in car traffic (Sugiyama et al., 2008). There experiments help to get insight into the behavior of individual pedestrians and cars. However, the laboratory experiments to study the behavior of bicycle flows are limited. Andresen, Chraibi, Seyfried, and Huber (2014) performed an experiment to observe basic cycling dynamics (accelerating, following) for the calibration and validation of 
a bicycle-following model. Seriani et al. (2015) organized an experimental study for estimating capacity of cycle lanes.

To the best of our knowledge, no laboratory experiment has been performed to investigate cyclist interaction behavior. The main question here boils down to whether these controlled experiments can be used to collect bicycle flow data under interacting situations and, more importantly, whether the resulting data are valid and useful. One might argue that people behave differently when they know that they are being observed. In general, cycling is considered to be less intuitive compared to walking, as people need to learn how to ride a bike. But for people who get used to cycle or learn how to cycle at their early ages, cycling might be as intuitive as walking. According to the three levels of cognitive control defined by Rasmussen (1986), cyclist behavior is assumed to be provoked by the so-called skill-based control of human body (other than rule-based and knowledge-based control). This type of behavior is provoked unconsciously and the decision is generally made at the spinal cord. Our main assumption here is that cyclists with certain experience cycle and behave when interacting with other cyclists with a relatively short decision process. Even if cyclists would notice that they are being observed, the cycling behavior is considered in natural circumstances. Moreover, during the experiment, participants are asked to perform the same cycling maneuvers a couple of times. Even though they know what to do, the actual performance of cyclists in each repetition would vary due to inter-personal and intra-personal variability. This justifies the applicability of a laboratory experiment for cyclist behavior research. However, one of the disadvantages of the laboratory experiment is that the learning effect can influence the participants' behavior as they watch and learn from other participants; hence the resulting data need to be assessed critically.

Moreover, there are various controlled variables to account for different cycling conditions in laboratory experiment. These controlled variables relate to distinct influencing factors on cycling maneuver, include social-economic characteristics of experiment participants (age, gender, and nationality), operational decisions (e.g., desired speed), types of bikes, directions of approaching, reaction on individuals or cyclist groups, level of familiarity, visual conditions, etc. Previous studies have looked into some of these aspects. For instance, Henderson and Lyons (1972) revealed gender differences in pedestrian motion, and this observation can be also found in human cycling behavior (Emond, Tang, \& Handy, 2009; Heesch, Sahlqvist, \& Garrard, 2012; Krizek, Johnson, \& Tilahun, 2005). Also a number of studies (Ma \& Luo, 2016; Schleinitz, Petzoldt, Franke-Bartholdt, Krems, \& Gehlert, 2017; Shepherd, 1994; Twaddle \& Grigoropoulos, 2016; Vansteenkiste, Cardon, D'Hondt, Philippaerts, \& Lenoir, 2013) looked into desired speed and acceleration profiles of cyclists under different circumstances. In literature, gender has been identified as an important factor influencing travel behavior, thus as a starting point gender differences in cycling interaction behavior will be examined in this study.

A controlled experiment to investigate cyclist behavior has been carried out at Delft University of Technology (TU Delft). Different control variables were specified, including different types of interactions, different cycling maneuvers, and single traveler versus a group of (two) cyclists. The resulting microscopic datasets can be used to study interaction behavior of cyclist flows, to calibrate and validate newly-developed cyclist models, to validate and develop new/improved theories of cyclist behavior, and to facilitate other related bicyclist research. This paper describes the controlled experimental design (research objectives and assumptions, measurement set-up, etc.), the resulting microscopic cyclist trajectory data, as well as preliminary results for one of the most common interaction situations: the bidirectional interaction between two individuals.

This paper is organized as follows. The proposed laboratory experiment is firstly described, with detailed experimental setup, scenario design, monitoring plan. Next, the procedure to derive microscopic cyclist trajectory data from video footages is presented. Then, preliminary results for the bidirectional 
interaction experiment are presented; both microscopic and macroscopic characteristics of bicycle flows are discussed. They are followed by conclusions and future research directions.

\section{Controlled experiment setup}

An envisaged conceptual model of cyclist interaction behavior should be able to describe how cyclists adjust their speeds, accelerations, steering angles and paths under different interacting situations. To develop such a model, a laboratory experiment has been performed to obtain data on individual cyclist interaction behavior. Ideally, this behavior is observed under various conditions, some of which have been identified in the previous section.

For this pilot experiment, we cannot cover all different conditions. As a simplification of reality and to meet basic requirement of the conceptual cyclist interaction model, three main control variables with 2 or 3 values were specified: a) type of interaction (face-to-face encounter, overtaking, crossing); b) acceleration behavior (continuous-speed cycling, acceleration, deceleration); and c) number of cyclists (single traveler versus a group of (two) cyclists). These control variables were selected to analyze the influence of the direction of approach, the acceleration condition, and the presence of groups on cycling interaction behavior. All bicycles were similar bikes (these bikes can be rented at train stations in the Netherlands, called OV-fiets), so only the behavior of the cyclist was observed, not the influence of the bike. TABLE 1 provides an overview of the experimental design with in total 13 scenarios, where only one variable is changed between scenarios. The comparison of trajectories between the unhindered and interacting cases would allow us to quantify the behavioral effects of interactions.

Table 1: Experimental scenario design

\begin{tabular}{|c|c|c|c|c|c|c|}
\hline Scenario No. & \multicolumn{3}{|l|}{ Unhindered maneuver type } & \multicolumn{3}{|c|}{ No. of cyclists } \\
\hline & Continuous-speed Cycling & \multicolumn{2}{|c|}{ Acceleration/Deceleration } & 1 person & \multicolumn{2}{|c|}{2 persons in paralle } \\
\hline 11 & $\mathrm{x}$ & & & $\mathrm{x}$ & & \\
\hline 12 & $\mathrm{x}$ & & & & $\mathrm{x}$ & \\
\hline 13 & & $\mathrm{x}$ & & $\mathrm{x}$ & & \\
\hline \multirow[t]{3}{*}{14} & & $\mathrm{x}$ & & & $\mathrm{x}$ & \\
\hline & \multicolumn{3}{|l|}{ Interaction type } & \multicolumn{3}{|c|}{ Group interaction type } \\
\hline & Bi-direction $\left(180^{\circ}\right)$ & Crossing $\left(90^{\circ}\right)$ & Overtaking $\left(0^{\circ}\right)$ & 1 on 1 & 1 on 2 & 2 on 2 \\
\hline 21 & $\mathrm{x}$ & & & $\mathrm{x}$ & & \\
\hline 22 & $\mathrm{x}$ & & & & $\mathrm{x}$ & \\
\hline 23 & $\mathrm{x}$ & & & & & $\mathrm{x}$ \\
\hline 31 & & $\mathrm{x}$ & & $\mathrm{x}$ & & \\
\hline 32 & & $\mathrm{x}$ & & & $\mathrm{x}$ & \\
\hline 33 & & $\mathrm{x}$ & & & & $\mathrm{x}$ \\
\hline 41 & & & $\mathrm{x}$ & $\mathrm{x}$ & & \\
\hline 42 & & & $\mathrm{x}$ & & $\mathrm{x}$ & \\
\hline 43 & & & $\mathrm{x}$ & & & $\mathrm{x}$ \\
\hline
\end{tabular}

In these experiments, twelve participants ( 7 males and 5 females which were university students) conducted the predefined maneuvers under unhindered and interacting situations of each scenario. In the unhindered situation, participants started, stopped, and cycled as they were instructed to do on a cycle path with a length of 40 meters, unhindered by other cyclists. Note that the acceleration and de- 
celeration maneuvers were conducted in the same run where participants were asked to first stop in the middle of the cycle path and then continue their trip to the end of the path. In the interacting situations, cyclists were overtaking, crossing or they had face-to-face encounters, in such a way that they had to interact to avoid collisions. Note that the length of 40 meters was considered to cover the entire interacting domain. To consider the heterogeneity between persons, twelve combinations of participants in each scenario were defined. And four repetitions/runs per combination of participants (in total 48 runs per scenario) were performed to get statistically significant results.

On the 10th of May in 2016, the controlled experiment was performed at TU Delft campus. This experiment took place at the intersection between the 'Berlageweg' bicycle path and the main bicycle path in front of the Faculty of Civil Engineering. An area of approximately 40 x 30 m covering the intersection was considered as the surveyed area (the camera monitoring area) (see FIGURE 1 (b)). No other traffic was allowed in the surveyed area. During the experiment, participants were instructed to ride down the middle of the bicycle path (the middle lane marking), so as to provoke their interaction with each other. They also started pedaling few meters beyond the boundary of the surveyed area so they entered the site with (random) initial speeds. Moreover, normal cycling behavior was expected and observed from the participants although the learning effect should be considered when analyzing the data. It was a cloudy day; hence the light condition was stable for data collection. The experiment was recorded with two video cameras with a visual angle of $60^{\circ}$ located above the experiment area. Since it was an outdoor environment, these two cameras were mounted to a portable pole of $15 \mathrm{~m}$ in height (see FIGURE 1 (a)). Given the constraints of the pole height and the camera vision angle, two video cameras were used to capture cyclist trajectories covering both sides of the surveyed area. In the future, this monitoring setup can be upgraded by increasing pole length and applying one high-resolution camera with a wide-angle lens to capture the entire area. Each participant and bike were equipped with identifiers (colored hats, number tabs, colored papers, etc.) for video capturing and monitoring purposes, so they could be recognized from video footage. The video clips were collected for all the 13 scenarios, with in total 624 individual experimental runs ( 13 scenarios $\mathrm{x} 12$ combinations of participants $\mathrm{x} 4$ repetitions). Advanced video processing software was used to derive microscopic trajectories from the video data; this is explained in details in the next section.

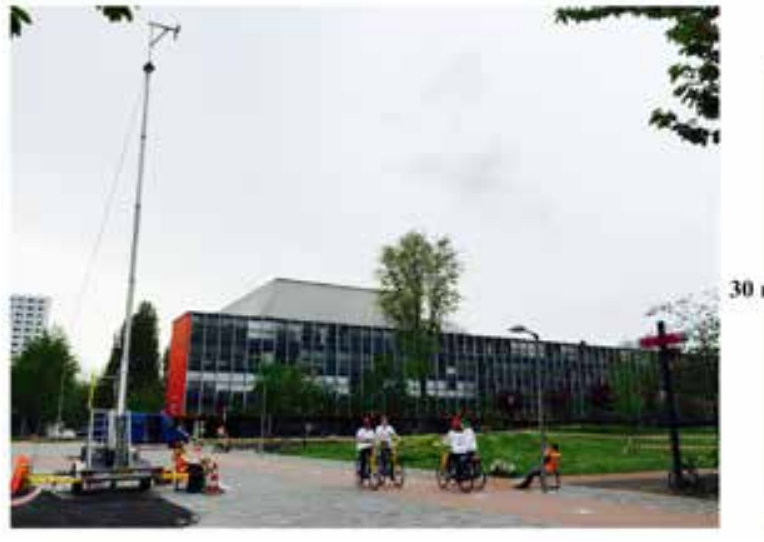

(a)

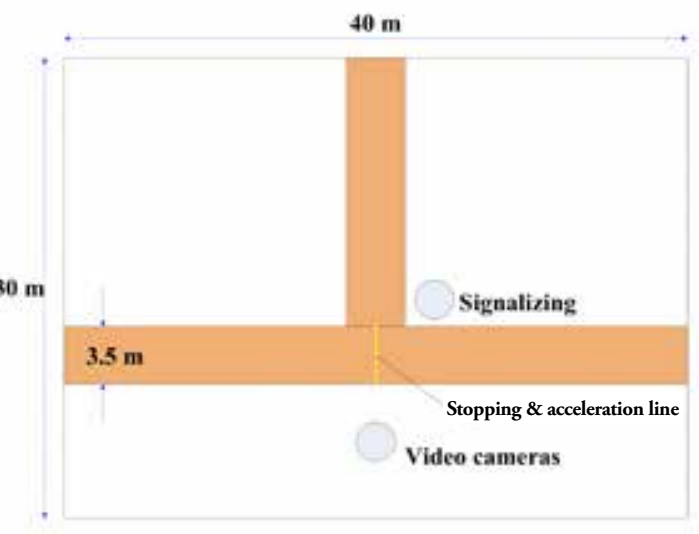

(b)

Figure 1: Overview of monitoring setup at the TU Delft campus: (a) a snapshot taken during the experiment; (b) a schematized aerial view of the experimental area monitored by two cameras 


\section{Microscopic trajectory dataset derivation}

Advanced video processing software/techniques have been used to obtain microscopic trajectory data. Specifically, the colored hats (red, green, yellow in this experiment), luggage carriers and two steering handlebars with colored papers (red, yellow, blue in this experiment) (see examples in FIGURE 2 (a, b)) can be identified by image processing tools to form useful trajectory data. For one individual participant, there are four detection/tracking points. We can derive various kinematic characteristics from the trajectory dataset of each point, including cyclist displacements, speeds, and accelerations in both longitudinal and lateral directions. The steering angle, defined as the angle between the longitudinal axis of a bicycle and the steered wheel direction, however, needs to be derived from all the four tracking positions. It is preferable that all participants are dressed in high-contrast colors regarding the signaling colors of the four positions to enable high detection quality. The detailed procedure is presented as follows:

1. The video clips are processed by a Moving Object Detection and Tracking (MODT) tool (Duives, Daamen, \& Hoogendoorn, 2012) to derive raw trajectory data. The tool automatically detects individual moving objects identified by different color ranges (in RGB code). However, the derived trajectory data might contain tracking errors (i.e., double counts and partial piecewise trajectories), which require a further cleaning process.

2. The raw trajectory data and all the videos (in the form of individual video stills) are read by a software package developed at TU Delft, called TrajectoryViewer. Using this tool, double counts can be removed, missing trajectory points can be added and partial trajectories can be combined. Since most of this work needs to be done manually, this step is the most timeconsuming one.

3. As the detected moving objects are extracted at different height levels, the processed trajectories need to be transformed in order to project various height positions to the ground level. A linear regression function is applied to the trajectory data derived from the TrajectoryViewer for height transformation. Examples in FIGURE 2 (c, d) show that the trajectories at the level of the head positions have been transformed to the ground level.

4. Next, Image Tracker (Knoppers, Van Lint, \& Hoogendoorn, 2012), a software developed at the TU Delft, is used to correct lens distortion of the two cameras (the barrel distortion in this case) and to obtain trajectories as seen from a top view instead of a side view. All the trajectories from the two cameras are transformed and scaled into a unified Cartesian coordinate system, under an orthorectification process.

5. The final step is to merge trajectories from the two cameras into consistent full trajectories that correspond to the same individual cyclists. As indicated in FIGURE 2 (e, f), there are overlapping trajectories in the middle part of the surveyed area, which need to be combined into connected trajectories, as shown in FIGURE 2 (g). Note that the trajectories in the middle part might not completely overlap, due to manual/software checking errors. In this work, two trajectories are merged at a predefined point in the horizontal direction. In the future, we will explain the use of a smoothing method to merge the trajectories. 


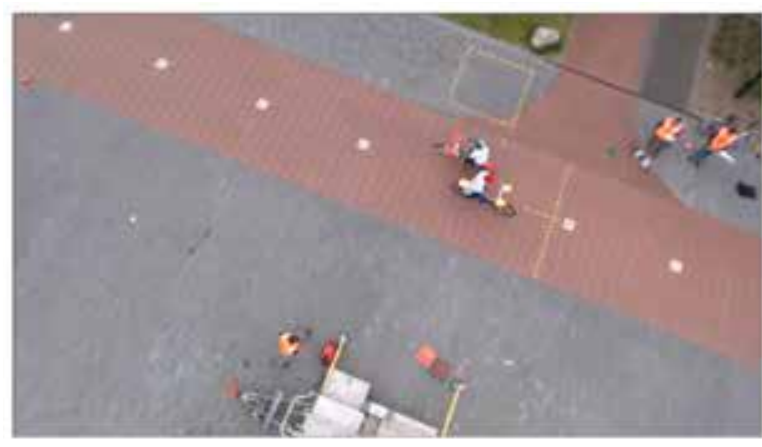

(a) One video still from the left camera

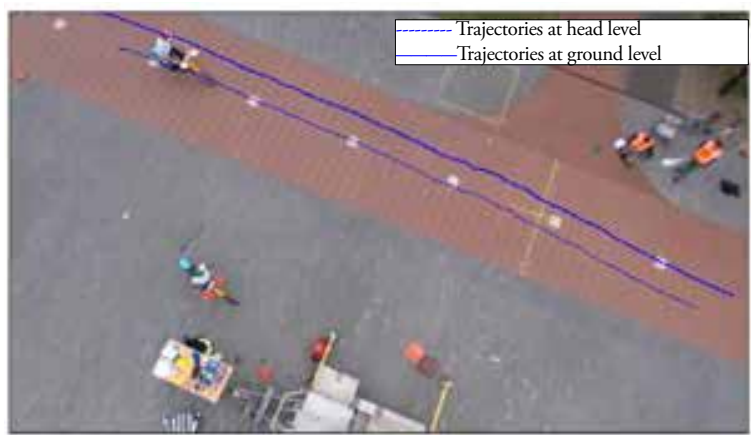

(c) Height transformation at the left camera

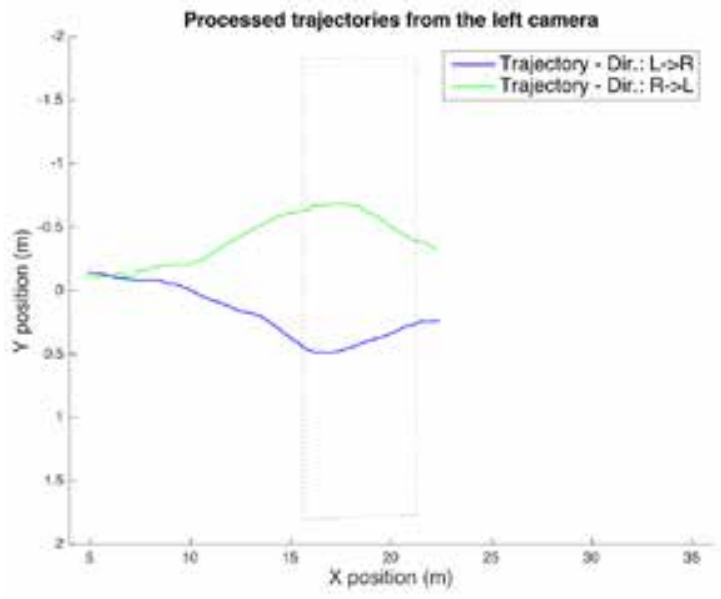

(e) Sample trajectories at the left camera

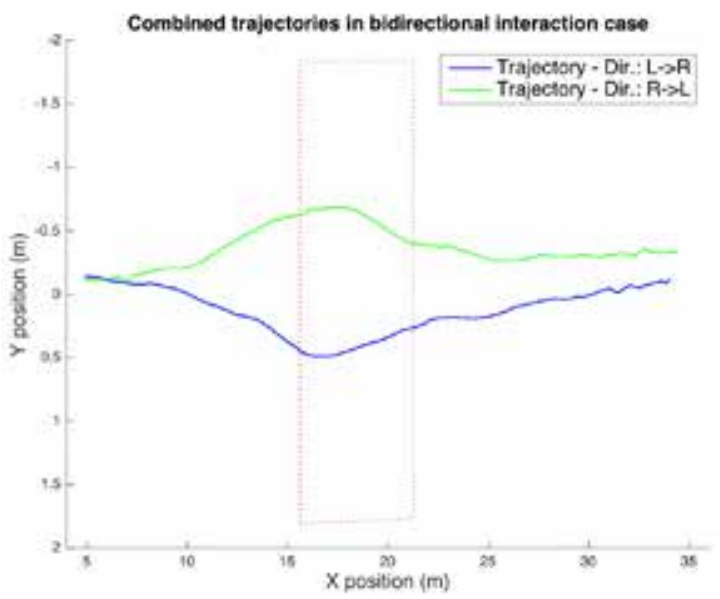

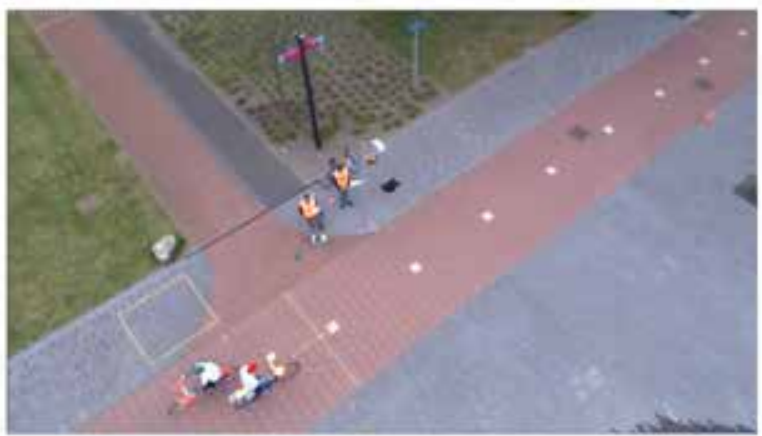

(b) One video still from the right camera

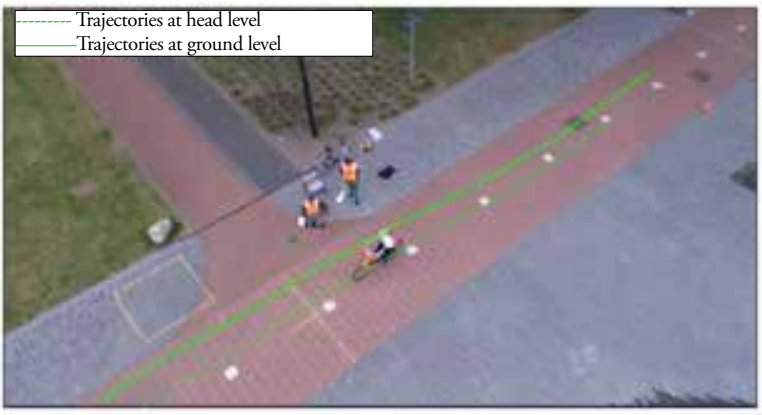

(d) Height transformation at the right camera

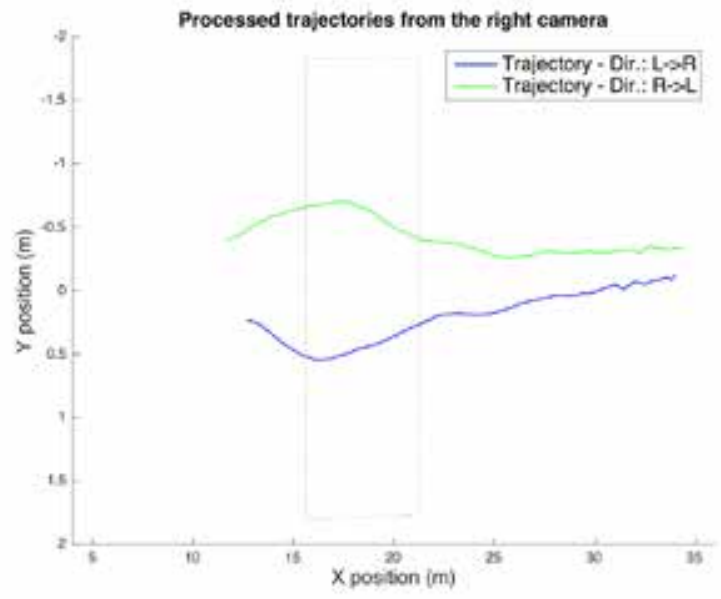

(f) Sample trajectories at the right camera

(g) Combined trajectories of the head positions projected to the ground

Figure 2: Examples of deriving trajectories from the two cameras (Dir.: direction; L: left; R: right) 


\section{$4 \quad$ Preliminary results on bidirectional interaction experiment}

We have first studied one of the most common interaction situations, namely the bi-directional cyclist interactions in a 1-on-1 relation, where the trajectory data of two of the scenarios: unhindered straightahead cycling (scenario 11) and bi-directional interaction in a 1-on-1 relation (scenario 21) have been extracted and analyzed. TABLE 2 provides an overview of the two analyzed scenarios, resulting in a dataset of 144 individual cyclist trajectories. The preliminary analysis focuses on investigating how cyclists change their paths and speeds in the interacting area. It is based only on the trajectories from the head positions (projected to the ground), which are sufficient to provide such information. The analysis of other aspects (such as accelerations and steering angles) will be addressed in future work.

Table 2: Overview of the considered scenarios in the preliminary analysis

\begin{tabular}{|l|l|l|l|l|}
\hline Scenario & $\begin{array}{l}\text { No. of combinations } \\
\text { of participants }\end{array}$ & No. of repetitions & No. of runs & No. of trajectories \\
\hline $\begin{array}{l}\text { Unhindered continuous-speed } \\
\text { cycling }\end{array}$ & 12 & 4 & 48 & 48 \\
\hline Bidirectional interaction & 12 & 4 & 48 & 96 \\
\hline
\end{tabular}

\subsection{Analysis objectives and assessment criteria}

In case of unhindered cycling, individual cycling capabilities and preferences determine the intended paths of cyclists in both lateral and longitudinal directions. Cycling outside of the intended path calculated in unhindered cycling situation is defined as interaction movement. In this work, these interaction movements have been identified. A simple measure to describe cyclist interaction behavior is the passing side, as participants may pass each other on either the left-hand side or the right-hand side. Interaction movements can be quantified by investigating the extent of evasion from the intended path in unhindered cycling. The effect of gender on cyclist interaction is also taken into account.

\subsection{Unhindered cycling}

The trajectories from the unhindered cycling case are used to derive boundaries for the intended paths, mean cycling paths (in lateral direction) and mean cycling speeds along the experimental path. The twelve participants cycled four times in the unhindered case. The observed average speeds of individual runs over each time instant (with complete trajectories) are shown in FIGURE 3. The t-test on the speed samples of the two genders rejects the null hypothesis that the speeds of male and female cyclists are not significantly different, at the $5 \%$ significance level. In general, men cycle faster than women, with an $8.5 \%$ higher average speed: $4.84 \mathrm{~m} / \mathrm{s}(17.4 \mathrm{~km} / \mathrm{h})$ versus $4.46 \mathrm{~m} / \mathrm{s}(16.1 \mathrm{~km} / \mathrm{h})$. Moreover, clear intravariability is observed from the trajectories of the same individuals. The experiment shows that both the speed and cycling path in each repetition are different and unique.

\subsection{Interaction movements}

It is found that individual cyclists make interaction-related maneuvers in almost all the runs of the bidirectional interaction scenario. These movements can be interpreted as some gallantry towards other cyclists. A clearly-visible evading (collision avoidance) maneuver is observed in the trajectory plot of two-cyclist face-to-face encounters in FIGURE 4. After passing the other cyclists, participants either stay 
in the deviated paths or move back toward the direction of the initial paths. The trajectories of the same individuals also vary in different repetitions, as observed in the unhindered case.

\subsection{Passing side}

In all the runs of the bidirectional interaction scenario, cyclists make an evading maneuver towards the right-hand side, except for one case where one participant moves to the left and collides with the incoming cyclist, who tries to make an evading maneuver towards his right-hand side. This observation can be explained by three reasons: the majority of the participants came from right-driving (cycling) countries; the experiment took place in a right-driving country; and the learning effect also played a role, as participants were watching how others performed. It is important to remark that in this experiment, both cyclists deviate from their intended paths; this is a mutual interaction procedure. This is not always the case in pedestrian bidirectional interactions (Huber et al., 2014), in which it has been observed that one pedestrian deviates and the other one stays in his/her intended path.

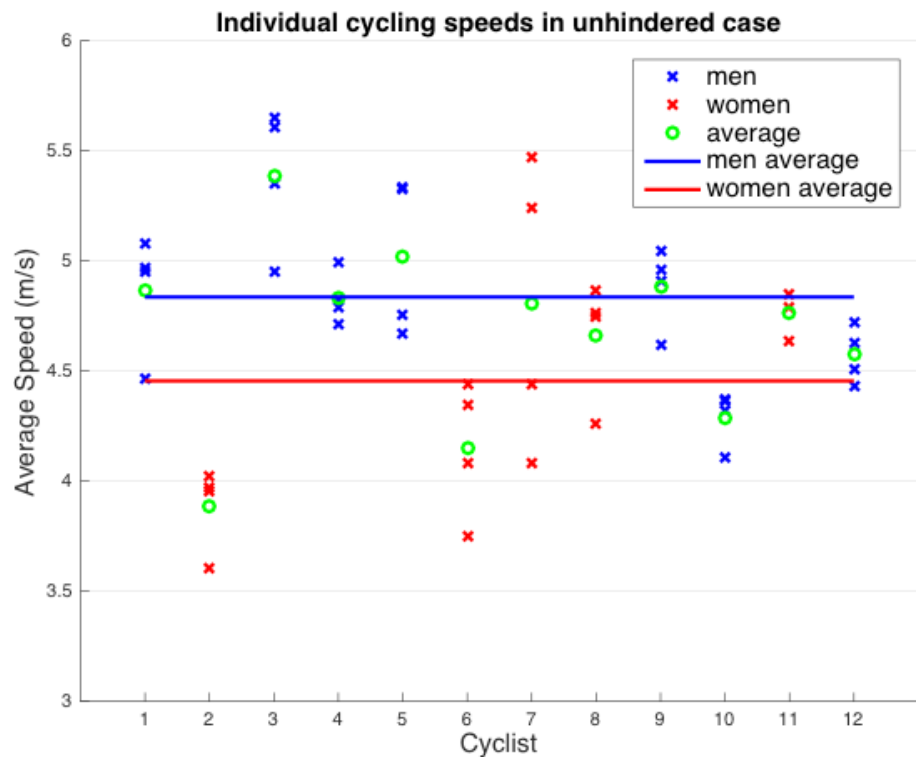

Figure 3: Cycling speeds in unhindered conditions

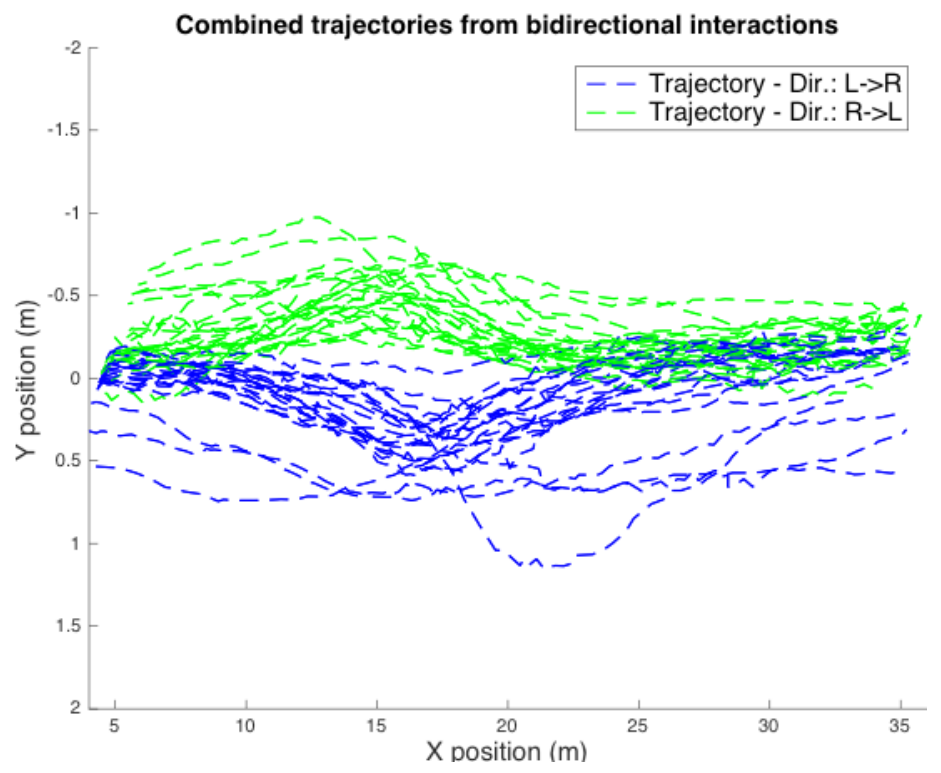


Figure 4: Combined trajectories in the bidirectional interaction cases

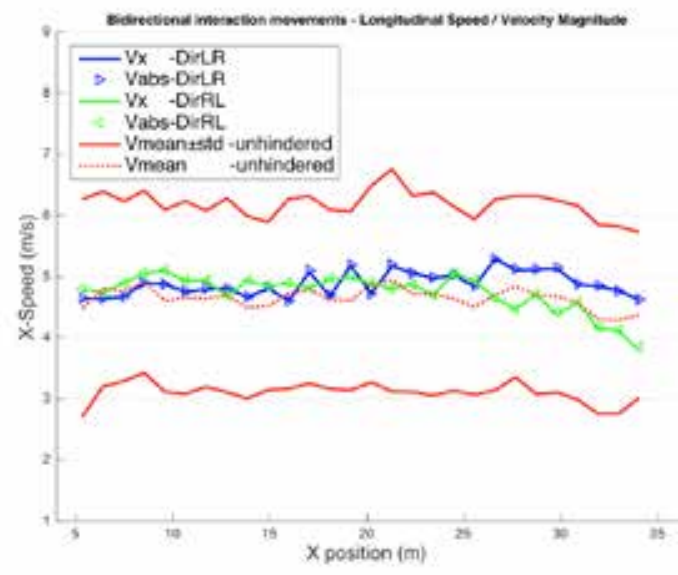

(a) Longitudinal/absolute speeds

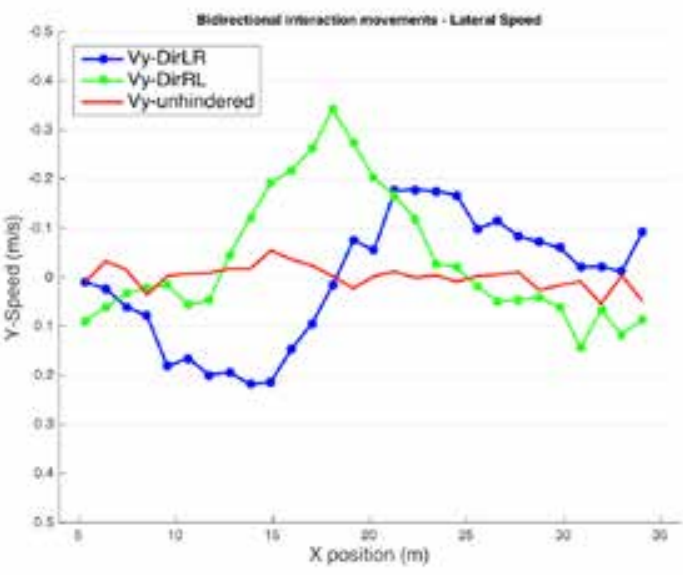

(b) Lateral speeds

Figure 5: Average longitudinal/absolute speeds (left) and average lateral speeds (right) of all individual cyclists in the bidirectional interaction cases ( $\mathrm{Vx}$ and $\mathrm{Vy}$ denote speeds in the longitudinal and lateral directions, respectively; Vabs depicts the velocity magnitude; Vmean is the average speed of all cyclists in the unhindered case.)
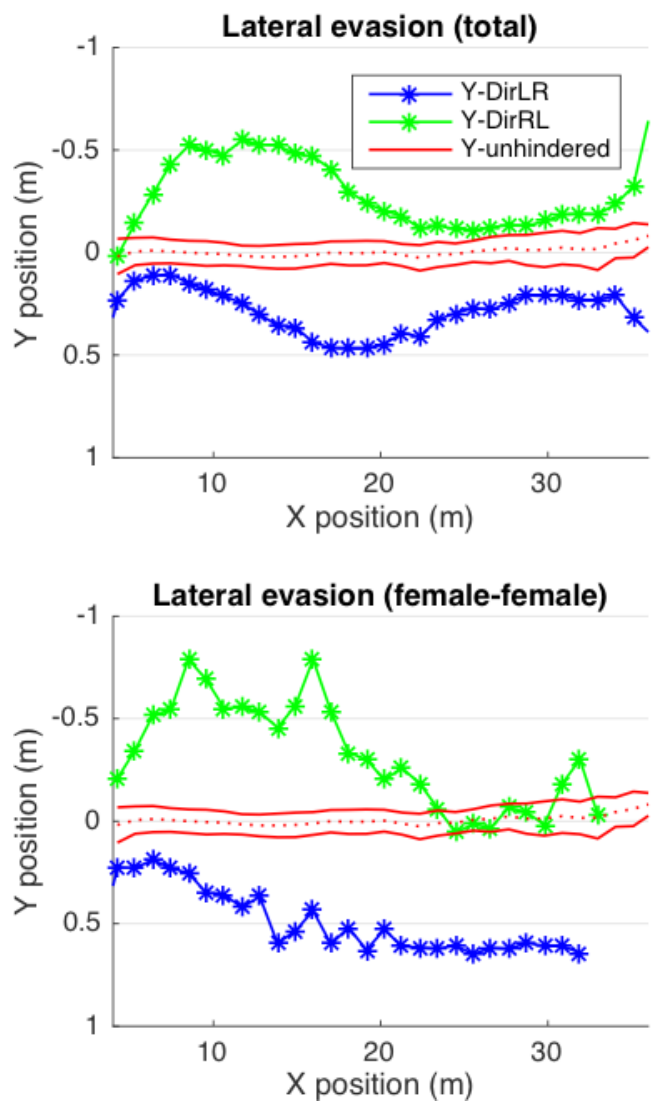
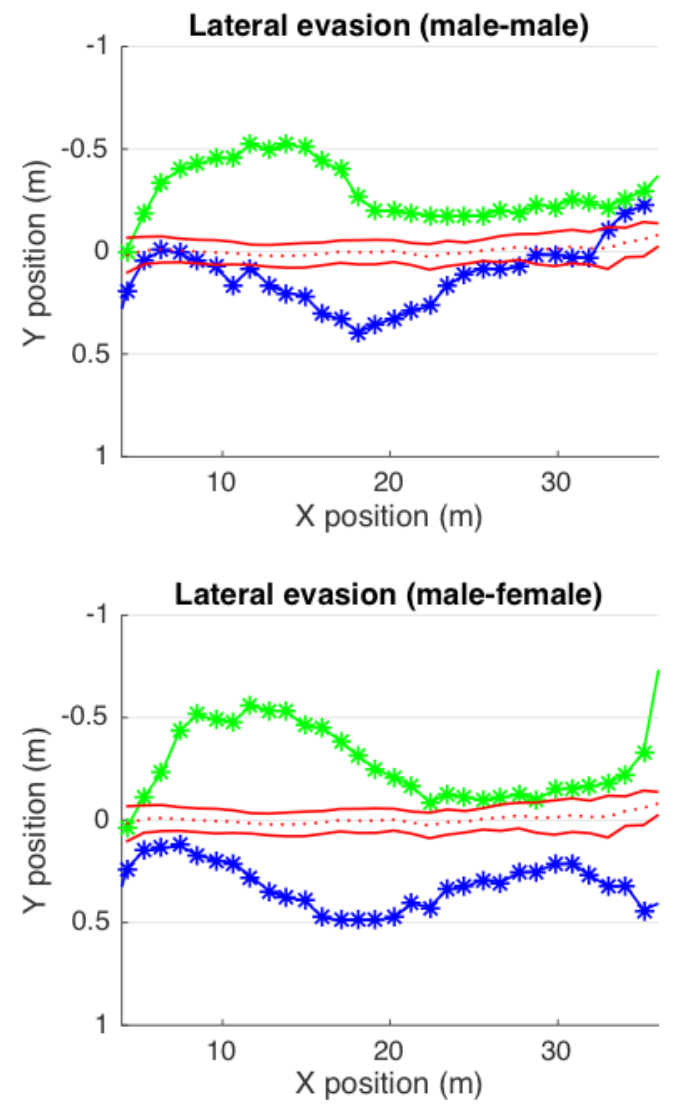
Figure 6: Average lateral deviation in displacement of different combinations of individual cyclists in the bidirectional interaction cases

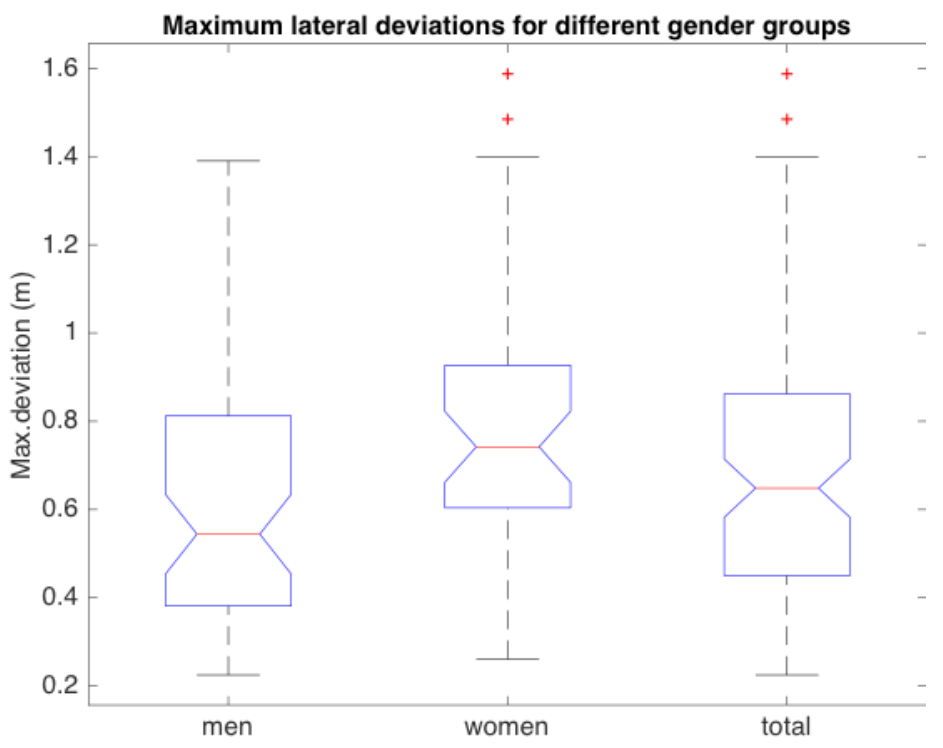

Figure 7: Maximum lateral deviation from the movement direction (the middle lane marking) of different gender groups in the bidirectional interaction scenario

\subsection{Extent of evasion}

In the case of a bidirectional interaction, cyclists need to move from their intended paths in order to avoid colliding with the incoming cyclists. We look at the change in both speeds and displacements. When encountering with another cyclist, there is no significant change in terms of speed in the longitudinal direction (Vx) and velocity magnitude (Vabs) (see FIGURE 5 (a)). However, obvious speed change is observed in the lateral direction $(\mathrm{Vy})$, because cyclists need to first turn to the right-hand side and then return to their original intended paths. FIGURE 5 (b) shows that the (maximum) lateral speeds in the two directions (sides) are of the same order.

Similarly, the distance deviation in the lateral direction is clearly observed at the interacting area. Cyclists start to deviate from their intended paths when they are around $30 \mathrm{~m}$ apart from each other. As they were instructed to cycle along the middle of the lane and to enter the site at their desired speeds, this indicates that the interaction (lateral deviation) took place within the range of the surveyed area (40 $\mathrm{m}$ in length). This observation also justifies the initial design for the length of the observation area.

Moreover, women laterally evade more than men in terms of distance, as shown in FIGURE 6. The maximum average amplitude of lateral evasion with male-on-male interactions is at the order of 1 $\mathrm{m}$, whereas this value is larger than $1 \mathrm{~m}$ in the case of female groups. And the t-test shows that the maximum lateral deviations in both distance and speed between genders are statistically different. The same observation can be seen in FIGURE 7, which shows that individual female cyclists have a larger maximum lateral deviation from the central line compared to male cyclists. This indicates that women keep larger lateral safety distances than men. Note the t-test reveals that men move faster than women in the lateral direction, which is in line with the observation in the unhindered case. However, the maximum lateral speeds do not have a direct relation with the maximum lateral evading distance.

The observation on gender differences can be related to the previous findings (Emond et al., 2009; 
Heesch et al., 2012; Krizek et al., 2005) that women reported more factors that influence the decision to use a bicycle (and the bicycling behavior in general). Previous evidence suggests that there exist gender differences in perception of bicycling safety. Our findings suggest that women are shown to give more courtesy when interacting with other cyclists. In general, women act more carefully than men in performing cycling maneuvers. The results reported here, however, need to be considered in light of some experiment constraints: a) the limited sample size; b) the learning effect. The results should be analyzed with caution. Nevertheless, the study reinforces the expectations and expands the knowledge obtained from literature on human cycling behavior at the operational level.

\section{$5 \quad$ Conclusions and future research}

This contribution presented a controlled laboratory experiment aimed to study cyclist behavior in unhindered cycling conditions and interaction situations. In total, 13 scenarios considering different types of interactions, acceleration profiles and group sizes have been carried out. Microscopic bicycle trajectories have been derived from video recordings of the experiment. A preliminary analysis of the trajectory data revealed how cyclists interact when they meet face-to-face another cyclist riding in the opposite direction.

Our findings confirm that men generally cycle faster than women. Both inter- and intra-variability in cycling speeds and paths are observed in the experiment. In cases of bidirectional interaction, cyclists strongly prefer moving towards their right-hand side. A clearly-visible evading (collision avoidance) maneuver is observed when two cyclists have face-to-face encounters, and this is a mutual interaction procedure where both cyclists deviate from their intended paths. When encountering the other cyclist, cyclists do not change speeds significantly in the longitudinal direction nor in absolute terms. However, a speed change is observed in the lateral direction. Cyclists start to deviate from their intended paths when they are around $30 \mathrm{~m}$ from each other. Moreover, women deviate more from their intended paths than men when they perform an evading maneuver.

In our future work, the video clips for all the remaining scenarios will be processed and analyzed. The cycling behavior under different interacting situations (including overtaking and crossing scenarios) will be investigated. We aim to put forward generic behavioral rules for cyclist interaction. In addition, the relation between distinct type of maneuvers and the related factors, such as the width of the lane, distance between cyclists, relative speeds and even eye contact, will be investigated. Note that the analysis presented in this paper is solely derived from the trajectories of head positions. There is abundant information hidden in the other three tracking positions (luggage carrier, right and left of the steering

bar). For instance, how cyclists react when encountering others regarding the operation of the steering handlebars. The answer can be found by studying the relations between steering angles and cycling trajectories. If the steering angles play a significant role in traffic operations, it may be necessary to include steering angles in microscopic cyclist simulation models. Moreover, the validation of these findings would require studies to be conducted in a wider context, considering socio-economic characteristics of cyclists and culture differences, and also considering empirical and/or experimental evidence from countries of large cyclist population, such as Norway, Sweden, China and India.

Knowing how cyclists interact with each other and the corresponding influential factors, and specially the gender differences would eventually contribute to better understand cycling behavior. All the findings serve as the foundation to develop sound and realistic behavioral theories and microscopic simulation models, which will help to design proper and safe cycling facilities, to evaluate investments on cyclist facilities, and to support policy development aimed at promoting cycling. 


\section{Acknowledgement}

This research was supported by the ALLEGRO project (Unravelling slow mode travelling and traffic: with innovative data to create a new transportation and traffic theory for pedestrians and bicycles), which is funded by the European Research Council (Grant Agreement No. 669792), and the Amsterdam Institute for Advanced Metropolitan Solutions. 


\section{References}

Amsterdam-Municipality, Jaarverslag. (2011). 2011 annual report. Amsterdam: Department of Infrstructure, Traffic and Transport.

Andresen, E., Chraibi, M., Seyfried, A., \& Huber, F. (2014). Basic driving dynamics of cyclists. In M. Behrisch, D. Krajzewicz, \& M. Weber (eds.), Simulation of urban mobility: First International Conference, SUMO 2013. Revised selected papers (p. 18-32). Berlin, Germany: Springer Berlin Heidelberg.

Botma, H., \& Papendrecht, H. (1991). Traffic operation of bicycle traffic. Transportation Research Record: Journal of the Transportation Research Board, 1320, 65-72.

Daamen, W., Hoogendoorn, S. P. (2003). Experimental research of pedestrian walking behavior. Transportation Research Record: Journal of the Transportation Research Board, 1828, 20-30.

Daamen, W., Hoogendoorn, S. P., Campanella, M., \& Versluis, D. (2012). Interaction behavior between individual pedestrians. In U. Weidmann, U. Kirsch, \& M. Schreckenberg (eds.), Pedestrian and evacuation dynamics (p. 1305-1314). Cham, Switzerland: Springer International Publishing.

Duives, D., Daamen, W., \& Hoogendoorn, S. P. (2012). Trajectory analysis of pedestrian crowd movements at a Dutch music festival. In U. Weidmann, U. Kirsch, \& M. Schreckenberg (eds.), Pedestrian and evacuation dynamics (p. 151-166). Cham, Switzerland: Springer International Publishing.

Emond, C., Tang, W., \&, Handy, S. (2009). Explaining gender difference in bicycling behavior. Transportation Research Record: Journal of the Transportation Research Board, 2125, 16-25.

Gould, G., \& Karner, A. (2009). Modeling bicycle facility operation: Cellular automaton approach. Transportation Research Record: Journal of the Transportation Research Board, 2140, 157-64.

Heesch, K. C., Sahlqvist, S., \& Garrard, J. (2012). Gender differences in recreational and transport cycling: A cross-sectional mixed-methods comparison of cycling patterns, motivators, and constraints. International Journal of Behavioral Nutrition and Physical Activity, 9(1), 106.

Henderson, L. F., \& Lyons, D. J. (1972). Sexual differences in human crowd motion. Nature, 240(5380), 353-355.

Homburger, W. S. (1976). Capacity of bus routes, and of pedestrian and bicycle facilities. Berkeley, CA: Institute of Transportation Studies.

Hoogendoorn, S. P., \& Daamen, W. (2016). Bicycle headway modeling and its applications. Proceedings of the Transportation Research Board 95th Annual Meeting, Washington DC.

Huber, M., Su, Y.-H., Krüger, M., Faschian, K., Glasauer, S., \& Hermsdörfer, J. (2014). Adjustments of speed and path when avoiding collisions with another pedestrian. PLoS ONE, 9(2), 1-13.

Knoppers, P., Van Lint, J. W. C., \& Hoogendoorn, S. P. (2012). Automatic stabilization of aerial traffic images. Proceedings of the Transportation Research Board 91st Annual Meeting, Washington, DC.

Krizek, K. J., Johnson, P. J., \& Tilahun, N. (2005). Gender differences in bicycling behavior and facility preferences. Research on Women's Issues in Transportation, 2, 31-40.

Ma, X., \& Luo, D. (2016). Modeling cyclist acceleration process for bicycle traffic simulation using naturalistic data. Transportation Research Part F: Traffic Psychology and Behavior, 40, 130-144.

Moussaï, M., Helbing, D., Garnier, S., Johansson, A., Combe, M, \& Theraulaz, G. (2009). Experimental study of the behavioral mechanisms underlying self-organization in human crowds. Proceedings of the Royal Society of London B: Biological Sciences, 276(1668), 2755-2762.

Navin, F. P. D. (1994). Bicycle traffic flow characteristics: Experimental results and comparisons. ITE Journal, 64(3), 31-37.

Raksuntorn, W., \& Khan, S. (2003). Saturation flow rate, start-up lost time, and capacity for bicycles at signalized intersections.. Transportation Research Record: Journal of the Transportation Research Board, 1852, 105-13. 
Rasmussen, J. (1986). Information processing and human-machine interaction: An approach to cognitive engineering. New York: Elsevier Science Inc.

Schleinitz, K., Petzoldt, T., Franke-Bartholdt, L., Krems, J. F., \& Gehlert, T. (2017). The German naturalistic cycling study-Comparing cycling speed of riders of different e-bikes and conventional bicycles. Safety Science, 92, 290-97.

Seriani, S., Fernandez, R., \& Hermosilla, E. (2015). Experimental study for estimating capacity of cycle lanes. Transportation Research Procedia, 8, 192-203.

Seyfried, A., Steffen, B., Passon, O., \& Klingsch, W. (2009). New insights into pedestrian flow through bottlenecks. Transportation Science, 43(3), 395-406.

Shepherd, R. (1994). Road and path quality for cyclists. Proceedings of the 17th ARRB Conference, Queensland, Australia.

Sugiyama, Y., Fukui, M., Kikuchi, M., Hasebe, K., Nakayama, A., Nishinari, K., Tadaki, S., \& Yukawa, S. (2008). Traffic jams without bottlenecks - experimental evidence for the physical mechanism of the formation of a jam. New Journal of Physics, 10(3), 033001.

Tian, W., Ma., J, Song, W. G., \& Liddle, J. (2012). Experimental study of pedestrian behaviors in a corridor based on digital image processing. Fire Safety Journal, 47, 8-15.

Twaddle, H., \& Grigoropoulos, G. (2016). Modeling the speed, acceleration, and deceleration of bicyclists for microscopic traffic simulation. Transportation Research Record: Journal of the Transportation Research Board, 2587, 8-16.

Vansteenkiste, P., Cardon, G., D'Hondt, E., Philippaerts, R., \& Lenoir, M. (2013). The visual control of bicycle steering: The effects of speed and path width. Accident Analysis \& Prevention, 51, 222-227.

Yang, J. M. (1985). Bicycle traffic in China. Transportation Quarterly, 39(1), 93-107.

Zamanov, M. (2012). Estimation of fundamental diagram of bicycle traffic flow using observations on bicycle lanes in Delft. Delft: Delft University of Technology. 